\title{
ON THE EXISTENCE OF OPTIMAL CONTROL FOR CONTROLLED STOCHASTIC PARTIAL DIFFERENTIAL EQUATIONS
}

\author{
NORIAKI NAGASE
}

\section{§1. Introduction}

In this paper we are concerned with stochastic control problems of the following kind. Let $Y(t)$ be a $d^{\prime}$-dimensional Brownian motion defined on a probability space $\left(\Omega, \mathscr{F}, \mathscr{F}_{t}, P\right)$ and $u(t)$ an admissible control. We consider the Cauchy problem of stochastic partial differential equations (SPDE in short)

$$
\left\{\begin{array}{l}
d p(t, x)=L(Y(t), u(t)) p(t, x) d t+M(Y(t)) p(t, x) d Y(t) \\
\quad x \in \mathbb{R}^{d}, t>0 \\
p(0, x)=\phi(x)
\end{array}\right.
$$

where $L(y, u)$ is the 2nd order elliptic differential operator and $M(y)$ the 1st order differential operator.

By a solution $p(t)=p^{u}(t)$, we mean $H^{1(\dagger)}$-valued $\mathscr{F}_{t}$-adapted process which satisfies

$$
\begin{aligned}
(p(t), \eta)= & (\phi, \eta)+\int_{0}^{t}\langle L(Y(s), u(s)) p(s), \eta\rangle d s \\
& +\int_{0}^{t}(M(Y(s)) p(s), \eta) d Y(s), \quad t \geq 0
\end{aligned}
$$

for any smooth $\eta$ where $\langle\cdot, \cdot\rangle$ is the pairing between $H^{-1}$ and $H^{1}$ and $(\cdot, \cdot)$ is $L^{2}\left(\mathbb{R}^{d}\right)$ inner product (see [4], [7]).

The SPDE (1.1) is related to the filtering, stochastic control with partial observation, population genetics etc. and investigated by Pardoux, Krylov \& Rozovskii and Rozovskii \& Shimizu, etc.

The purpose of this paper is to prove the existence of optimal controls for the following problem. Define a criterion $J(u)$ by

Received November 21, 1987.

(†) $H^{l}=H^{l}\left(\mathbb{R}^{d}\right)$ denotes the Sobolev space $W_{2}^{l}\left(\mathbb{R}^{d}\right), l=0, \pm 1, \cdots$. 


$$
J(u)=E\left[F\left(p^{u}\right)+G\left(p^{u}(T)\right)\right]
$$

where $F$ and $G$ are real valued functions on $L^{2}\left(0, T ; L^{2}\left(\mathbb{R}^{d}\right)\right)$ and $L^{2}\left(\mathbb{R}^{d}\right)$ respectively. Now we want to minimize $J(u)$ by a suitable choice of an admissible process $u$.

In Section 2 we will recall some known results in our convenient way and formulate our problem precisely. In Section 3 we will prove that the solution $p^{u}$ depends on $u$ continuously which derives the existence of optimal control [Theorem 3.2]. In Section 4 we apply our results to stochastic control with partial observation, where an observation noise may depend on a state noise.

The author wishes to thank Professor M. Nisio for her valuable suggestion and encouragement and the referee for many helpful comments.

\section{$\S 2$. Notation and preliminaries}

We assume the following conditions (A.1) (A.3).

$$
\begin{aligned}
& \text { b: } \mathbb{R}^{d} \times \mathbb{R}^{d^{\prime}} \longrightarrow \mathbb{R}^{d} \otimes \mathbb{R}^{L} \\
& \sigma: \mathbb{R}^{d} \times \mathbb{R}^{d^{\prime}} \longrightarrow \mathbb{R}^{d} \otimes \mathbb{R}^{d^{\prime}} \\
& \text { a: } \mathbb{R}^{d} \times \mathbb{R}^{d^{\prime}} \longrightarrow \mathbb{R}^{d} \otimes \mathbb{R}^{d} \\
& h: \mathbb{R}^{d} \times \mathbb{R}^{d^{\prime} \longrightarrow \mathbb{R}^{d^{\prime}}}
\end{aligned}
$$

are bounded and continuous and $a$ is symmetric.

(A.2) There exists $\delta>0$ such that

$$
2 a(x, y)-3 \sigma(x, y) \sigma^{*}(x, y) \geq \delta I \quad \text { for any }(x, y) \in \mathbb{R}^{d} \times \mathbb{R}^{d^{\prime}}
$$

where $\sigma^{*}$ is the transposed matrix of $\sigma$.

$$
\begin{aligned}
& a(\cdot, y), \sigma(\cdot, y) \text { are } C^{\hat{m}+1} \text {-class in } x \in \mathbb{R}^{d}, \\
& h(\cdot, y), b(\cdot, y) \text { are } C^{\hat{m}} \text {-class in } x \in \mathbb{R}^{d},
\end{aligned}
$$

and their derivatives are bounded and continuous in $(x, y) \in \mathbb{R} \times \mathbb{R}^{d^{\prime}}$, where $\hat{m}=\max \{2, m\}$ and $m$ is a given nonnegative integer.

Let $\Gamma$ be a convex and compact subset of $\mathbb{R}^{L}$.

Definition 2.1. $\mathscr{A}=(\Omega, \mathscr{F}, P, Y, u)$ is called an admissible system, if $(\Omega, \mathscr{F}, P)$ is a probability space and $u$ is a $\Gamma$-valued measurable process and $Y$ is a $d^{\prime}$-dimensional $\left(\mathscr{F}_{t}\right)$-Brownian motion on $(\Omega, \mathscr{F}, P)$, where $\mathscr{F}_{t}=\sigma\left\{Y(s), \int_{0}^{s} u(\tau) d \tau ; s \leq t\right\}$. 
$\mathfrak{A}$ denotes the totality of admissible systems.

For $\mathscr{A} \in \mathfrak{A}, \pi^{\mathscr{\alpha}}$ denotes the image measure of $(Y, u)$ on $C\left(0, T ; \mathbb{R}^{d^{\prime}}\right) \times$ $L^{2}(0, T ; \Gamma)$.

Endowing the uniform topology on $C\left(0, T ; \mathbb{R}^{a^{\prime}}\right)$ and the weak topology on $L^{2}(0, T ; \Gamma)$, we have

Lemma 2.1. $\left\{\pi^{\mathscr{d}} ; \mathscr{A} \in \mathfrak{Q}\right\}$ is compact under the Prokhorov metric. (See Fleming \& Pardoux [2] Lemma 2.3.)

Define $L(y, u) \in \mathscr{L}\left(H^{1}, H^{-1}\right), M^{k}(y) \in \mathscr{L}\left(H^{1}, L^{2}\left(\mathbb{R}^{d}\right)\right)\left(k=1, \cdots, d^{\prime}, y \in \mathbb{R}^{d^{\prime}}\right.$, $u \in \Gamma$ ) by

$$
\begin{gathered}
\langle L(y, u) p, q\rangle=-\sum_{i, j=1}^{d}\left(a_{i j}(\cdot, y) \frac{\partial p}{\partial x_{i}}, \frac{\partial q}{\partial x_{j}}\right)+\sum_{j=1}^{d}\left(\tilde{b}_{j}(\cdot, y, u) p, \frac{\partial q}{\partial x_{j}}\right) \\
\left(M^{k}(y) p, \eta\right)=-\sum_{i=1}^{d}\left(\sigma_{i k}(\cdot, y) \frac{\partial p}{\partial x_{i}}, \eta\right)+\left(\tilde{h}_{k}(\cdot, y) p, \eta\right)
\end{gathered}
$$

for $p, q \in H^{1}$ and $\eta \in L^{2}\left(\mathbb{R}^{d}\right)$, where $(\cdot, \cdot)=$ the inner product in $L^{2}\left(\mathbb{R}^{d}\right)$, $\langle\cdot, \cdot\rangle=$ the duality pairing between $H^{-1}$ and $H^{1}$ and

$$
\begin{aligned}
& \tilde{b}_{j}(x, y, u)=\sum_{i=1}^{L} b_{j l}(x, y) u_{l}-\sum_{i=1}^{d} \frac{\partial a_{i j}}{\partial x_{i}}(x, y) \\
& \tilde{h}_{k}(x, y)=h(x, y)-\sum_{i=1}^{d} \frac{\partial \sigma_{i k}}{\partial x_{i}}(x, y) .
\end{aligned}
$$

By (A.1) (A.3), there exists $\alpha>0$ and $\lambda \in \mathbb{R}$ such that

$$
\begin{array}{r}
-2\langle L(y, u) p, p\rangle+\lambda\|p\|_{0}^{2} \geq \alpha\|p\|_{1}^{2}+3 \sum_{k=1}^{d^{\prime}}\left\|M^{k}(y) p\right\|_{0}^{2} \\
\text { for any } p \in H^{1}, y \in \mathbb{R}^{d}, u \in \Gamma
\end{array}
$$

where $\|\cdot\|_{l}=$ the $H^{l}$-norm $(l=0, \pm 1, \cdots)$ (for the proof, see $\S 2$ of Krylov \& Rozovskii [4]).

(2.3) is called the coercivity condition.

For an admissible system $\mathscr{A}=(\Omega, \mathscr{F}, P, Y, u)$, putting $L^{\mathscr{\alpha}}(t)=L(Y(t), u(t))$ and $M^{\star k}(t)=M^{k}(Y(t))$, we consider the Cauchy problem of SPDE on $(\Omega, \mathscr{F}, P)$,

$$
\left\{\begin{array}{c}
d p(t)=L^{\mathscr{s}}(t) p(t) d t+M^{\mathscr{s}}(t) p(t) d Y(t) \\
\quad t>0 \\
p(0)=\phi \in H^{\hat{m}}
\end{array}\right.
$$

where $M^{\star}(t)=\left(M^{\star 1}(t), \cdots, M^{\varkappa d^{\prime}}(t)\right)$. 
Definition 2.2. By a solution of SPDE (2.4), we mean an $H^{1}$-valued $\mathscr{F}_{t}$-adapted process $p(t)$ defined on $(\Omega, \mathscr{F}, P)$ such that

(1) $E\left[\int_{0}^{T}\|p(t)\|_{1}^{2} d t\right]<\infty$

(2) for any $\eta \in H^{1}$ and $t \in[0, T]$

$$
(p(t), \eta)=(\phi, \eta)+\int_{0}^{t}\left\langle L^{\star}(s) p(s), \eta\right\rangle d s+\int_{0}^{t}\left(M^{\star}(s) p(s), \eta\right) d Y(s)
$$

holds.

By the coercivity condition (2.3), we have the following proposition. (See [5], [7].)

Proposition 2.1. For each $\mathscr{A} \in \mathfrak{A}$, the equation (2.4) has a unique solution $p=p^{\star}$ which satisfies

$$
p \in L^{2}\left((0, T) \times \Omega ; H^{\hat{n}+1}\right) \cap L^{2}\left(\Omega ; C\left(0, T ; H^{\hat{n}}\right)\right)
$$

and

$$
\begin{aligned}
\|p(t)\|_{0}^{2}= & \|\phi\|_{0}^{2}+2 \int_{0}^{t}\left\langle L^{\star}(s) p(s), p(s)\right\rangle d s \\
& +2 \int_{0}^{t}\left(M^{\star}(s) p(s), p(s)\right) d Y(s)+\int_{0}^{t}\left\|M^{\star} p(s)\right\|_{0}^{2} d s .
\end{aligned}
$$

The solution $p=p^{\star}$ of the SPDE (2.4) is called the response for $\mathscr{A}$.

Remark 2.1. We can apply the results of Pardoux [7] also to the triplet $\left(V, H, V^{*}\right)$, where $V=H^{l+1}, H=H^{l}$ and $V^{*}=H^{l-1}(l=0,1, \cdots, \hat{m})$. Define $\tilde{L}(y, u) \in \mathscr{L}\left(H^{l+1}, H^{l-1}\right), \tilde{M}(y) \in \mathscr{L}\left(H^{l+1}, H^{l}\right)$ similarly to $L(y, u), M(y)$, where we replace $\langle\cdot, \cdot\rangle$ and $(\cdot, \cdot)$ by " $\langle\cdot, \cdot\rangle_{l}=$ the duality pairing between $H^{l-1}$ and $H^{\imath+1}$ " and " $(\cdot, \cdot)_{l}=$ the inner product in $H^{\imath "}$ repsectively in (2.1), (2.2). Then the coercivity condition holds. (In (2.3), $\|\cdot\|_{0}$ and $\|\cdot\|_{1}$ are replaced by $\|\cdot\|_{l}$ and $\|\cdot\|_{l+1}$ respectively.) Appealing to Krylov \& Rozovskii [4], the solution $p$ of (2.4) turns out a unique solution of SPDE (2.9)

$$
\left\{\begin{array}{c}
d p(t)=\tilde{L}(Y(t), u(t)) p(t) d t+\tilde{M}(Y(t)) p(t) d Y(t) \\
t>0 \\
p(0)=\phi .
\end{array}\right.
$$

Moreover $p(t)$ satisfies similar equality to (2.8). (i.e. " 0 " is replaced by “ $l$ ".)

Let $F: L^{2}\left(0, T ; H^{m+1}\right) \rightarrow \mathbb{R}$ and $G: H^{m} \rightarrow \mathbb{R}$ be weakly continuous functions. 
For $\mathscr{A} \in \mathfrak{A}$, we define the pay-off function $J(\mathscr{A})$ by

$$
J(\mathscr{A})=E\left[F\left(p^{\star}\right)+G\left(p^{\star}(T)\right)\right] .
$$

We want to minimize its value by a suitable choice of $\mathscr{A} \in \mathfrak{A}$.

\section{§3. Existence of optimal control}

First of all we will prove that the solution $p^{\infty}$ of (2.4) depends on $\mathscr{A}$ continuously.

THEOREM 3.1. If $\pi^{\star s(n)} \rightarrow \pi^{\star}$ in law, then

$$
p^{\star(n)} \longrightarrow p^{\infty} \text { in law as } L^{2}\left(0, T ; H^{m+1}\right) \text {-random variable }
$$

and

$$
p^{\infty(n)}(T) \longrightarrow p^{\infty}(T) \text { in law as } H^{m} \text {-random variable, }
$$

where we endow the weak topologies on $L^{2}\left(0, T ; H^{m+1}\right)$ and $H^{m}$.

For the proof we need the following two lemmas.

Lemma 3.1. There exists a constant $K>0$ such that

$$
\begin{aligned}
& E\left\{\int_{0}^{T}\left\|p^{s}(t)\right\|_{i+1}^{2} d t\right\} \leq K\|\phi\|_{l}^{2} \\
& E\left\{\sup _{0 \leq t \leq T}\left\|p^{s}(t)\right\|_{l}^{2}\right\} \leq K\|\phi\|_{l}^{2} \\
& E\left\{\int_{0}^{T}\left\|p^{s}(t)\right\|_{l}^{4} d t\right\} \leq K\|\phi\|_{l}^{4} \\
& \quad \text { for any } \mathscr{A} \in \mathfrak{A} . \quad(l=0,1, \cdots, \hat{m}) .
\end{aligned}
$$

According to [6] we introduce the spaces $\mathscr{H}_{r}(D)$ and $\mathscr{H}_{r}(T, D)$ as follows. Set $\hat{\psi}(\cdot, x)=$ the Fourier transformation in $t$ of $\psi(\cdot, x),\|\cdot\|_{2, D}=$ the $H^{2}(D)$-norm and $\|\cdot\|_{*}=$ the norm of the dual space $\left(H^{2}(D)\right)^{*}$, where we identify $H^{1}(D)$ with its dual space.

$$
\mathscr{H}_{r}(D)=\left\{\psi \in L^{2}\left(-\infty, \infty ; H^{2}(D)\right) ; \int_{-\infty}^{\infty}|\tau|^{2 \tau}\|\hat{\psi}(\tau)\|_{*}^{2} d \tau<\infty\right\}
$$

where

$$
\begin{gathered}
\|\psi\|_{\mathscr{P}_{\gamma}(D)}=\left\{\int_{-\infty}^{\infty}\|\psi(t)\|_{2, D}^{2} d t+\int_{-\infty}^{\infty}|\tau|^{2 \tau}\|\hat{\psi}(\tau)\|_{*}^{2} d \tau\right\}^{1 / 2} \\
\mathscr{H}_{\gamma}(T, D)=\left\{\left.\psi\right|_{[0, T]} ; \psi \in \mathscr{H}_{\gamma}(D)\right\}
\end{gathered}
$$

where 


$$
\|\psi\|_{\mathscr{P}_{r}(T, D)}=\inf \left\{\|\varphi\|_{\mathscr{P}_{r}(D)} ; \varphi(t)=\psi(t) \text { a.e. on }[0, T]\right\} \text {. }
$$

Remark 3.1. If $D$ is a bounded and open subset of $\mathbb{R}^{d}$ with a smooth boundary, then, by the compactness lemma ([6] p. 60) the imbedding: $\mathscr{H}_{\gamma}(T, D) \rightarrow L^{2}\left(0, T ; H^{1}(D)\right)$ is compact.

Lemma 3.2. Let $0<\gamma<1 / 4$, then for each $\mathscr{A} \in \mathfrak{A}$,

$$
p^{\star} \in \mathscr{H}_{r}(T, D) \text { a.s. }
$$

and there exists $K>0$ such that

$$
E\left[\left\|p^{\mathscr{S}}\right\|_{\mathscr{P}_{r}(T, D)}^{2}\right] \leq K\|\phi\|_{2}^{2} \quad \forall \mathscr{A} \in \mathfrak{A} .
$$

Proof of Lemma 3.1. (3.3) and (3.4) are easy variants of Corollary 2.2 of Krylov \& Rozovskii [4]. Now we will show (3.5). Since the response $p$ is the solution of (2.9), using Itô's formula, we get

$$
\begin{aligned}
\|p(t)\|_{l}^{4}= & \|\phi\|_{l}^{4}+4 \int_{0}^{t}\|p(s)\|_{l}^{2}\langle\tilde{L}(s) p(s), p(s)\rangle_{l} d s \\
& +2 \int_{0}^{t}\|p(s)\|_{l}^{2}\|\tilde{M}(s) p(s)\|_{l}^{2} d s+4 \sum_{k=1}^{d^{\prime}} \int_{0}^{t}\left(\tilde{M}^{k}(s) p(s), p(s)\right)_{l}^{2} d s \\
& +4 \int_{0}^{t}\|p(t)\|_{l}^{2}(\tilde{M}(s) p(s), p(s))_{l} d Y(s)
\end{aligned}
$$

where $\tilde{L}(t)=\tilde{L}(Y(t), u(t))$ and $\tilde{M}(t)=\tilde{M}(Y(t))$.

Hence, using the coercivity condition, we have

$$
\begin{aligned}
E\left[\|p(t)\|_{l}^{4}\right]-\|\phi\|_{l}^{4}= & 2 E\left[\int_{0}^{t}\|p(s)\|_{l}^{2}\left\{2\langle\tilde{L}(s) p, p\rangle_{l}+\|\tilde{M}(s) p\|_{l}^{2}\right\} d s\right] \\
& +4 E\left[\int_{0}^{t} \sum_{k=0}^{d^{\prime}}(\tilde{M}(s) p, p)_{l}^{2} d s\right] \\
\leq & 2 E\left[\int_{0}^{t}\|p(s)\|_{l}^{2}\left\{\lambda^{\prime}\|p(s)\|_{l}^{2}-\alpha^{\prime}\|p(s)\|_{l+1}^{2}\right\} d s\right] \\
\leq & 2 \lambda^{\prime} E\left[\int_{0}^{t}\|p(s)\|_{l}^{4} d s\right] .
\end{aligned}
$$

So the Gronwall's inequality derives (3.5).

Proof of Lemma 3.2. For the convenience, we extend $p(t)$ on $(-\infty, \infty)$ in the following way

$$
\begin{aligned}
p(t) & =p(t), t \in[0, T] \\
& =0, \quad t \in(-\infty, \infty) \backslash[0, T] .
\end{aligned}
$$

Since $p(t)$ is a solution of (2.9), applying Itô's formula, we obtain 


$$
\begin{aligned}
2 \pi i \tau(\hat{p}(\tau), \eta)_{2}= & (\phi, \eta)_{2}-(p(T), \eta)_{2} \exp \{-2 \pi i \tau T\} \\
& +\langle\hat{\tilde{L} p}(\tau), \eta\rangle_{2}+\int_{0}^{T} \exp \{-2 \pi i \tau t\}(\tilde{M}(t) p, \eta)_{2} d Y(t)
\end{aligned}
$$

for any $\eta \in H^{3}$.

Let $\left\{\eta_{k}\right\}_{k \geq 1}$ be an orthonormal basis in $H^{3}$. Using (3.3), (3.4) and (3.9), we have

$$
4 \pi^{2} \tau^{2} E\left[\|\hat{p}(\tau)\|_{1}^{2}\right]=4 \pi^{2} \tau^{2} \sum_{k=1}^{\infty} E\left\{\left|\left(\hat{p}(\tau), \eta_{k}\right)_{2}\right|^{2}\right\} \leq K_{1}\|\phi\|_{2}^{2}+K_{2} E\left[\|\widehat{\tilde{L} p}(\tau)\|_{1}^{2}\right] .
$$

Let $0<\gamma<1 / 4$ and $0<\kappa<3 / 2$, then

$$
\begin{aligned}
& \int_{-\infty}^{\infty} E\left\{|\tau|^{2 \gamma}\|\hat{p}(\tau)\|_{*}^{2}\right\} d \tau \leq \int_{|\tau| \leq 1} E\left[\|\hat{p}(\tau)\|_{1}^{2}\right] d \tau+\int_{|\tau| \geq 1} E\left[\frac{2|\tau|^{2}}{1+|\tau|^{k}}\|\hat{p}(\tau)\|_{1}^{2}\right] d \tau \\
& \quad \leq K_{3}\left\{E\left[\int_{-\infty}^{\infty}\|p(t)\|_{1}^{2} d t\right]+\int_{-\infty}^{\infty} \frac{d \tau}{1+|\tau|^{k}}\|\phi\|_{1}^{2}+E\left[\int_{-\infty}^{\infty}\|\tilde{L}(t) p\|_{1}^{2} d t\right]\right\} \\
& \quad \leq K_{4}\|\phi\|_{2}^{2} .
\end{aligned}
$$

This concludes the lemma.

Remark 3.2. (3.5) implies the uniform integrability of

$$
\int_{0}^{T}\left\|p^{\mathscr{A}}(t)\right\|_{l}^{2} d t, \quad \mathscr{A} \in \mathfrak{A} .
$$

Remark 3.3. We define the metric $d$ on $H=L^{2}\left(0, T ; H^{m+1}\left(\mathbb{R}^{d}\right)\right)$ by

$$
d(p, q)=\sum_{k=1}^{\infty} \frac{1}{2^{k}} \min \left(\left|\left(e_{k}, p-q\right)\right|, 1\right\} \quad p, q \in H
$$

where $(\cdot, \cdot)$ is the inner product on $H$ and $\left\{e_{k}\right\}_{k=1}^{\infty}$ is the orthonormal basis on $H$. Then Lemma 3.1 and Prokhorov's theorem imply that the totality of image measure $p^{\star}(\mathscr{A} \in \mathfrak{U})$ is relatively compact as a set of measures on the metric space $(H, d)$.

On the other hand, on each bounded set of $H$ the weak topology is metrizable by the metric $d$. Therefore, for any weakly closed set $F$ of $H, F \cap\{q \in H ;\|q\| \leq r\}(r>0)$ is closed with respect to the metric $d$.

Under this observation, $\left\{p^{\star} ; \mathscr{A} \in \mathfrak{X}\right\}$ is relatively compact as a set of measures on $H$ associated with the weak topology.

Proof of Theorem 3.1. Let $D_{k}(k=1,2, \cdots)$ be bounded and open subsets of $\mathbb{R}^{d}$ with smooth boundary, $\overline{D_{k}} \subset D_{k+1}$ and $\bigcup_{k=1}^{\infty} D_{k}=\mathbb{R}^{d}$. For an admissible system $\mathscr{A}=(\Omega, \mathscr{F}, P, Y, u)$, 


$$
\begin{aligned}
& \mu^{\infty}=\text { the image measure of }\left(Y, u, p^{\star}\right) \text { on } S, \\
& \mu_{k}^{\alpha}=\text { the image measure of }\left(Y, u, p^{\star}\right) \text { on } S_{k}
\end{aligned}
$$

where

$$
S=C\left(0, T ; \mathbb{R}^{d^{\prime}}\right) \times L^{2}(0, T ; \Gamma) \times L^{2}\left(0, T ; H^{m+1}\left(\mathbb{R}^{d}\right)\right),
$$

and

$$
S_{k}=C\left(0, T ; \mathbb{R}^{d^{\prime}}\right) \times L^{2}(0, T ; \Gamma) \times L^{2}\left(0, T ; H^{1}\left(D_{k}\right)\right)
$$

endowing the weak topology on $L^{2}\left(0, T ; H^{m+1}\left(\mathbb{R}^{d}\right)\right)$ and the strong topology on $L^{2}\left(0, T ; H^{1}\left(D_{k}\right)\right)$. By the compactness of $\left\{\pi^{\mathscr{A}} ; \mathscr{A} \in \mathfrak{A}\right\}$ and Remark 3.3, $\mathfrak{P}=\left\{\mu^{\star s} ; \mathscr{A} \in \mathfrak{A}\right\}$ is relatively compact. Moreover, by Lemma 3.2 and Remark 3.1, $\mathfrak{\Re}_{k}=\left\{\mu_{k}^{\infty} ; \mathscr{A} \in \mathfrak{X}\right\}$ is relatively compact.

Hence there exist a subsequence $\left\{\mathscr{A}\left(n^{\prime}\right)\right\}_{n^{\prime}}$, a probability $\mu$ on $S$ and a probability $\mu_{k}$ on $S_{k}(k=1,2, \cdots)$ such that

$$
\mu^{\infty\left(n^{\prime}\right)} \longrightarrow \mu \text { in law as } n^{\prime} \longrightarrow \infty
$$

and

$$
\mu_{k}^{\infty\left(n^{\prime}\right)} \longrightarrow \mu_{k} \text { in law as } n^{\prime} \longrightarrow \infty .
$$

By Skorohod's theorem, we can construct the $S_{k}$-valued random variables $\left(Y_{n^{\prime}}, u_{n^{\prime}}, p_{n^{\prime}}\right),(Y, u, p), n^{\prime}=1,2, \cdots$, on a probability space $(\Omega, \mathscr{F}, P)$ such that

(3.14) the law of $\left(Y_{n^{\prime}}, u_{n^{\prime}}, p_{n^{\prime}}\right)=\mu_{k}^{\alpha\left(n^{\prime}\right)}, n^{\prime}=1,2, \cdots$,

(3.15) the law of $(Y, u, p)=\mu_{k}$

and

(3.16) $\left(Y_{n^{\prime}}, u_{n^{\prime}}, p_{n^{\prime}}\right) \longrightarrow(Y, u, p)$ almost surely $\left(n^{\prime} \longrightarrow \infty\right)$

as $S_{k}$-valued random variables.

Now we will prove the following lemma.

Lemma 3.3. Let $\psi:[0, T] \rightarrow \mathbb{R}$ be an absolutely continuous function with $\psi^{\prime} \in L^{2}(0, T)$ and $\psi(T)=0$ and $\eta \in C_{0}^{\infty}\left(\mathbb{R}^{d}\right)$ with $\operatorname{supp}(\eta) \subset D_{k}$, then $(Y, u, p)$ of (3.16) satisfies

$$
\begin{aligned}
(\phi, \eta) \psi(0) & +\int_{0}^{T} \psi^{\prime}(t)(p(t), \eta) d t+\int_{0}^{T} \psi(t)\langle L(Y(t), u(t)) p, \eta\rangle d t \\
& +\int_{0}^{T} \psi(t)(M(Y(t)) p, \eta) d Y(t)=0 .
\end{aligned}
$$


Proof. Since $p_{n^{\prime}}$ is the solution of the $\operatorname{SPDE}(2.4)$ for $\left(Y_{n^{\prime}}, u_{n^{\prime}}\right)$, using Itô's formula to (2.5), we get

$$
\begin{aligned}
(\phi, \eta) \psi(0) & +\int_{0}^{T} \psi^{\prime}(t)\left(p_{n^{\prime}}(t), \eta\right) d t+\int_{0}^{T} \psi(t)\left\langle L\left(Y_{n^{\prime}}(t), u_{n^{\prime}}(t)\right) p_{n^{\prime}}, \eta\right\rangle d t \\
& +\int_{0}^{T} \psi(t)\left(M\left(Y_{n^{\prime}}(t)\right) p_{n^{\prime}}, \eta\right) d Y_{n^{\prime}}(t)=0 .
\end{aligned}
$$

By Remark 3.2 and (3.16), we get

$$
E\left[\int_{0}^{T}\left\|p_{n^{\prime}}(t)-p(t)\right\|_{1, D_{k}}^{2} d t\right] \longrightarrow 0 \quad\left(n^{\prime} \rightarrow \infty\right)
$$

Recalling "supp $(\eta) \subset D_{k}$ ", we obtain

$$
\begin{aligned}
& \int_{0}^{T} \psi(t)\left\langle L\left(Y_{n^{\prime}}(t), u_{n^{\prime}}(t)\right) p_{n^{\prime}}, \eta\right\rangle d t \\
& \quad \longrightarrow \int_{0}^{T} \psi(t)\langle L(Y(t), u(t)) p, \eta\rangle d t \quad \text { in } L^{2}(\Omega) . \\
& \psi(t)\left(p_{n^{\prime}}(t), \eta\right) \longrightarrow \psi(t)(p(t), \eta) \quad \text { in } L^{2}([0, T] \times \Omega)
\end{aligned}
$$

and

$$
\psi(t)\left(M\left(Y_{n^{\prime}}(t)\right) p_{n^{\prime}}, \eta\right) \longrightarrow \psi(t)(M(Y(t)) p, \eta) \quad \text { in } L^{2}([0, T] \times \Omega) .
$$

For the proof of (3.19), putting

$$
\begin{aligned}
& q_{n^{\prime}}(t)=\psi(t)\left(b_{i l}\left(\cdot, Y_{n^{\prime}}(t)\right) p_{n^{\prime}}(t), \eta\right) \\
& q(t)=\psi(t)\left(b_{i l}(\cdot, Y(t)) p(t), \eta\right)
\end{aligned}
$$

and $u(t)=\left(u^{1}(t), \cdots, u^{L}(t)\right)$, we have

$$
\begin{gathered}
\int_{0}^{T} \psi(t)\left(b_{i l}\left(\cdot, Y_{n^{\prime}}(t)\right) p_{n^{\prime}}(t), \eta\right) u_{n^{\prime}}^{l}(t) d t-\int_{0}^{T} \psi(t)\left(b_{i l}(\cdot, Y(t)) p(t), \eta\right) u^{l}(t) d t \\
=\int_{0}^{T} u_{n^{\prime}}^{l}(t)\left(q_{n^{\prime}}(t)-q(t)\right) d t+\int_{0}^{T}\left(u_{n^{\prime}}^{l}(t)-u^{l}(t)\right) q(t) d t
\end{gathered}
$$

By (3.18), the 1st term of the right hand side of (3.22) converges to 0 in $L^{2}(\Omega)$. By Remark 3.2 and (3.16), we get

$$
E\left[\left\{\int_{0}^{T}\left(u_{n^{\prime}}^{l}(t)-u^{l}(t)\right) q(t) d t\right\}^{2}\right] \longrightarrow 0
$$

This implies (3.19). (3.20) and (3.21) can be proved similarly. Moreover, combining (3.21) with (3.16), we get

$$
\begin{aligned}
\int_{0}^{T} \psi(t) & \left.\left(M\left(Y_{n^{\prime}}(t)\right) p_{n^{\prime}}, \eta\right) d Y_{n^{\prime}}, t\right) \\
& \longrightarrow \int_{0}^{T} \psi(t)(M(Y(t)) p, \eta) d Y(t) \quad \text { in } L^{2}(\Omega) .
\end{aligned}
$$


Hence, by taking limit of (3.17) ${ }_{n^{\prime}}$, we obtain (3.17).

Let $i_{k}: S \rightarrow S_{k}$ be the canonical injection. Then by the definition

$$
i_{k}\left(\mu^{\star\left(n^{\prime}\right)}\right)=\mu_{k}^{\infty\left(n^{\prime}\right)} \text { and } i_{k}(\mu)=\mu_{k} .
$$

Let $(\tilde{Y}, \tilde{u}, \tilde{p})$ be $S$-valued random variable whose law $=\mu . \quad$ Then (3.25) implies that the law of $\left(\tilde{Y}, \tilde{u},\left.\tilde{p}\right|_{D_{k}}\right)=\mu_{k}$.

Hence, by Lemma 3.3, $\left(\tilde{Y}, \tilde{u},\left.\tilde{p}\right|_{D_{k}}\right)$ satisfies the equation (3.17). Noting that $\operatorname{supp}(\eta) \subset D_{k}$, we obtain

$$
\begin{aligned}
(\phi, \eta) \psi(0) & +\int_{0}^{T} \psi^{\prime}(t)(\tilde{p}(t), \eta) d t+\int_{0}^{T} \psi(t)\langle L(\tilde{Y}(t), \tilde{u}(t)) \tilde{p}, \eta\rangle d t \\
& +\int_{0}^{T} \psi(t)(M(\tilde{Y}(t)) \tilde{p}, \eta) d \tilde{Y}(t)=0 .
\end{aligned}
$$

Since $k$ is arbitrary, (3.26) holds for any $\eta \in C_{0}^{\infty}\left(\mathbb{R}^{d}\right)$.

By the same argument as Theorem 1.3 in [7], $\tilde{p}$ becomes a solution of $\operatorname{SPDE}(2.4)$ for $(\tilde{Y}, \tilde{u})$. Since the law of $(\tilde{Y}, \tilde{u})=\pi^{s}$, we get

$$
\mu=\text { the law of }(\tilde{Y}, \tilde{u}, \tilde{p})=\mu^{\infty} .
$$

This means that any convergent subsequence of $\left\{\mu^{\alpha\left(n^{\prime}\right)}\right\}$ converges to $\mu^{\star}$. Hence the original sequence $\left\{\mu^{\star(n)}\right\}$ converges to $\mu^{\star}$. So we get (3.1). Next we consider the law of $\left(Y, u, p^{\star}, p^{\alpha}(T)\right)$ then by the similar argument we can prove (3.2).

THEOREM 3.2. If $F$ and $G$ are bounded from below, then there exists an optimal admissible system $\tilde{\mathscr{A}} \in \mathfrak{U}$ that is

$$
\inf \{J(\mathscr{A}) ; \mathscr{A} \in \mathfrak{X}\}=J(\tilde{\mathscr{A}}) .
$$

Proof. By theorem 3.1,

$$
J_{n}(\mathscr{A})=E\left[\min \left\{F\left(p^{\star}\right), n\right\}+\min \left\{G\left(p^{\star}(T)\right), n\right\}\right]
$$

is continuous on $\mathfrak{A}$. Since $J(\mathscr{A})$ is the limit function of non-decreasing sequence $\left\{J_{n}(\mathscr{A})\right\}_{n=1}^{\infty}$, it is lower-semicontinuous on $\mathfrak{A}$. This concludes the theorem.

\section{§4. Optimal control for partially observed diffusions}

In this section we will apply Theorem 3.2 to the stochastic control problems for partially observed diffusions where an observation noise may depend on a state noise.

We assume the following conditions (A.4) (A.6). 
(A.4) $\quad \hat{\sigma}: \mathbb{R}^{d} \times \mathbb{R}^{d^{\prime}} \longrightarrow \mathbb{R}^{d} \otimes \mathbb{R}^{d}$ is bounded and continuous

(A.5) There exists $\delta>0$ such that

$$
\hat{\sigma}(x, y) \hat{\sigma}^{*}(x, y)-2 \sigma(x, y) \sigma^{*}(x, y) \geq \delta \mathrm{I} \quad \text { for } \forall(x, y) \in \mathbb{R}^{d} \times \mathbb{R}^{d}
$$

(A.6) $\hat{\sigma}(\cdot, y)$ is $C^{3}$-class in $x \in \mathbb{R}^{d}$ and all derivatives are bounded and continuous in $(x, y) \in \mathbb{R}^{d} \times \mathbb{R}^{d}$.

Put $a(x, y)=\left(\hat{\sigma}(x, y) \hat{\sigma}^{*}(x, y)+\sigma(x, y) \sigma^{*}(x, y)\right) / 2$, then $a(x, y)$ and $\sigma(x, y)$ satisfy (A.2).

Now we will consider the optimal control problems of the following kind. Let $X(t)$ denote the state process being controlled, $Y(t)$ the observation process and $u(t)$ the control process. The state and observation processes are governed by the stochastic differential equations

$$
\left\{\begin{aligned}
d X(t) & =b(X(t), Y(t)) u(t) d t+\hat{\sigma}(X(t), Y(t)) d \hat{W}(t)+\sigma(X(t), Y(t)) d W(t) \\
X(0) & =\xi
\end{aligned}\right.
$$

and

$$
\left\{\begin{aligned}
d Y(t) & =h(X(t)) d t+d W(t) \\
Y(0) & =0
\end{aligned}\right.
$$

where $\hat{W}$ and $W$ are independent Brownian motions with values in $\mathbb{R}^{d}$ and $\mathbb{R}^{d^{\prime}}$ respectively on a probability space $(\Omega, \mathscr{F}, \hat{P})$.

The problem is to minimize a criterion of the form

$$
J(u)=\hat{E}\left[\int_{0}^{T} f(X(t)) d t+g(X(T))\right] .
$$

In the customary version of stochastic control under partial observation, $u(t)$ is a function of the observation process $Y(s), s \leq t$. Instead of discussing the problem of this type, we treat some wider class of admissible controls inspired by Fleming \& Pardoux [2].

Let

$$
\rho(t)=\exp \left\{\int_{0}^{t} h(X(s)) d Y(s)-\frac{1}{2} \int_{0}^{t}|h(X(s))|^{2} d s\right\} .
$$

Then $\hat{W}$ and $Y$ become independent Brownian motions under a new probability $P$ defined by

$$
d P=\rho(T)^{-1} d \hat{P}
$$

and $X(t)$ becomes a solution of the following SDE 


$$
\left\{\begin{aligned}
d X(t)= & \{b(X(t), Y(t)) u(t)-\sigma(X(t), Y(t)) h(X(t))\} d t \\
& +\hat{\sigma}(X(t), Y(t)) d \hat{W}(t)+\sigma(X(t), Y(t)) d Y(t) \\
X(0)= & \xi .
\end{aligned}\right.
$$

Suppose $\xi$ has a probability density $\phi \in H^{2}\left(\mathbb{R}^{d}\right)$.

Definition 4.1. $\mathscr{A}=(\Omega, \mathscr{F}, P, \hat{W}, Y, u, \xi)$ is called an admissible system, if

(1) $(\Omega, \mathscr{F}, P)$ is a probability space

(2) $u$ is $\Gamma$-valued measurable process

(3) $Y$ is a $d^{\prime}$-dimensional $\left(\mathscr{F}_{t}\right)$-Brownian motion where

$$
\mathscr{F}_{t}=\sigma\left\{Y(s), \int_{0}^{s} u(\tau) d \tau ; s \leq t\right\}
$$

(4) $\hat{W}$ is a $d$-dimensional Brownian motion

(5) $\xi$ is a $d$-dimensional random variable and its distribution has the density $\phi$

(6) $\xi, \hat{W}$ and $(Y, u)$ are independent with respect to $P$.

For an admissible system $\mathscr{A}$, the solution $X(t)=X^{\mathscr{s}}(t)$ of the SDE (4.6) is called the response for $\mathscr{A}$. Putting $d \hat{P}=\rho(T) d P$, we define the pay-off function by

$$
J(\mathscr{A})=\hat{E}\left[\int_{0}^{T} f\left(X^{\mathscr{\alpha}}(t)\right) d t+g\left(X^{\mathscr{\omega}}(T)\right)\right]
$$

where $f, g \in L^{2}\left(\mathbb{R}^{d}\right)$ and non-negative.

By the similar argument as Rozovskii [8], we obtain the following.

Proposition 4.1. Let $p^{\star s}$ be a solution of the SPDE (2.4) for an admissible system $\mathscr{A}$, then $p^{\star}(t)$ is the unnormalized conditional density of $X^{\star}(t)$ with respect to $\mathscr{F}_{t}$. Namely, for every $\varphi \in L^{\infty}\left(\mathbb{R}^{d}\right), t \in[0, T]$

$$
E\left[\varphi\left(X^{\star s}(t)\right) \rho(t) \mid \mathscr{F}_{t}\right]=\left(\varphi, p^{\alpha}(t)\right) P \text {-a.s. }
$$

holds, where $(\cdot, \cdot)$ is the inner product in $L^{2}\left(\mathbb{R}^{d}\right)$.

Using (4.8), we get

$$
J(\mathscr{A})=E\left[\int_{0}^{T}\left(f, p^{\infty}(t)\right) d t+\left(g, x^{\infty}(T)\right)\right] .
$$

Since $\left(f, p^{s}(t)\right)$ and $\left(g, p^{s}(T)\right)$ are non-negative, Theorem 3.2 assures the existence of an optimal admissible system. Namely, 
THEOREM 4.1. There exists an optimal admissible system $\tilde{\mathscr{A}}$, that is

$$
\inf _{\mathscr{A}: \text { ad.sys. }} J(\mathscr{A})=J(\tilde{\mathscr{A}}) \text {. }
$$

\section{REFERENCES}

[1] A. Bensoussan, Maximum principle and dynamic programming approaches of the optimal control of partially observed diffusions, Stochastics, 9 (1983), 169-222.

[2] W. H. Fleming and E. Pardoux, Optimal control for partially observed diffusions, SIAM J. Control Optim., 20 (1982), 261-285.

[ 3 ] N. Ikeda and S. Watanabe, Stochastic Differential Equations and Diffusion Processes, Kodansha/North-Holland, Tokyo/Amsterdam, 1981.

[4] N. V. Krylov and B. L. Rozovskii, On the Cauchy problem for stochastic partial differential equations, Math. USSR-Izv., 11 (1977), 1267-1284.

[5] — On the conditional distributions of diffusion processes, Math. USSR-Izv., 12 (1978), 336-356.

[6 ] J. L. Lions, Equations Différentielles Opérationnelles et Problèmes aux Limites, Springer-Verlag, Berlin, 1961.

[7] E. Pardoux, Stochastic partial differential equations and filtering of diffusion processes, Stochastics, 3 (1979), 127-167.

[8] B. L. Rozovskii, Nonnegative $L^{1}$-solutions of second order stochastic parabolic equations with random coefficients, Transl. Math. Eng., (1985), 410-427.

[ 9 ] B. L. Rozovskii and A. Shimizu, Smoothness of solutions of stochastic evolution equations and the existence of a filtering transition density, Nagoya Math. J., 84. (1981), 195-208.

Department of Mathematics and System Fundamentals

Division of System Science

Kobe University

Rokko, Kobe, 657

Japan 